\title{
Assessment of intratumor immune-microenvironment in colorectal cancers with extranodal extension of nodal metastases
}

Matteo Fassan ${ }^{1 *+}\left(0\right.$, Luca Vianello $^{1 \dagger}$, Diana Sacchi ${ }^{1 \dagger}$, Giuseppe N. Fanelli ${ }^{1}$, Giada Munari ${ }^{1}$, Marco Scarpa $^{2}$, Rocco Cappellesso ${ }^{1}$, Fotios Loupakis ${ }^{3}$, Cristiano Lanza ${ }^{1}$, Roberta Salmaso ${ }^{1}$, Claudia Mescoli ${ }^{1}$, Nicola Valeri ${ }^{4,5}$, Marco Agostini $i^{2,6,7}$, Edoardo D'Angelo², Sara Lonardi ${ }^{3}$, Salvatore Pucciarelli ${ }^{4}$, Nicola Veronese ${ }^{8,11}$, Claudio Luchini ${ }^{9}$ and Massimo Rugge ${ }^{1,10}$

\begin{abstract}
Background: No data is available on the molecular background of the extra-nodal extension (ENE) of lymph node metastasis (LN) in colorectal cancer (CRC).

Methods: A series of 22 ENE-positive CRCs was considered and three samples per case were selected (the primary $C R C$, an ENE-negative and an ENE-positive metastatic $L N)$. Samples $(n=66)$ were analysed by immunohistochemistry for PD-L1, CD4, CD8, CD68 and CD80. Fifteen out of twenty-two cases were further profiled through a hotspot multigene mutational custom panel, including 164 hotspot regions of AKT1, APC, BRAF, CTNNB1, KIT, KRAS, NRAS, PDGFRA, PIK3CA, PTEN and TP53 genes.

Results: A significantly higher percentage of CD4-, CD8- and CD68-positive cells was observed at the invasive front of both CRCs and in ENE in contrast with what observed at the core of both CRCs and their matched nodal metastases. ENE was also characterized by a significantly higher number of CD80-positive cells. No significant difference was observed in PD-L1 distribution among the different specimens. Fourteen out of 15 CRCs (93\%) showed at least a driver mutation. The most frequently mutated gene was TP53 $(n=8$ tumors), followed by APC $(n=6), \operatorname{BRAF}(n=4)$, KRAS, NRAS and PIK3CA $(n=2)$. In 11 out of 15 CRCs $(73 \%)$ the mutational profiling of the primary tumor was consistent with what obtained from the two matched LNs.
\end{abstract}

Conclusions: A heterogeneous intratumor immune-microenvironment has been observed in ENE-positive CRCS, which are characterized by an increased leukocytic infiltration at the ENE invasive front.

Keywords: Extranodal extension, Metastasis, Colorectal cancer, Biomarkers

\footnotetext{
*Correspondence: matteo.fassan@unipd.it

${ }^{\dagger}$ Matteo Fassan, Luca Vianello and Diana Sacchi contributed equally to

this work

${ }^{1}$ Surgical Pathology \& Cytopathology Unit, Department of Medicine

(DIMED), University of Padua, via Gabelli 61, 35121 Padua, Italy

Full list of author information is available at the end of the article
} 


\section{Background}

Colorectal cancer (CRC) is the third more frequent cancer and the fourth cause of cancer death all over the world [1].

CRC survival is mainly dependent upon the tumornode-metastasis (TNM) stage of disease at diagnosis $[1,2]$. However, not all the patients, who have the same stage, show comparable prognoses. Therefore, further research is needed to identify novel prognostic factors for the stratification of risk of recurrence in patients classified with the same stage of disease.

Recently, the extra-nodal extension (ENE) of nodal metastasis has emerged as a promising and independent prognostic factor in several epithelial malignancies [3-14].

From the pathological point of view, ENE is different from free tumor deposits in the fibroadipose tissue, even if both conditions present similar phenotypic features. In contrast to what is observed in ENE, tumor deposits do not present residual nodal tissue and are considered as a separate entity in the tumor-node-metastasis (TMN) staging system, having a specific category into the $\mathrm{N}$ group (i.e. N1c) [15]. On the other hand, ENE, which is defined by an invasion of cancer cells/glands from an intra-nodal metastasis through the lymph node capsule, is still not considered in the TNM system as a prognostic parameter in nodal-metastatic CRC patients.

In a recent meta-analysis from our group, specifically designed on the prognostic role of ENE in CRC [16], we demonstrated the importance of such parameter in influencing the survival of CRC patients. Notably, 611 patients out of the analysed nodal-positive 1336 (45.7\%), showed an ENE-positive status, Indicating that ENE is a frequent condition among CRC metastatic tumors.

The invading potential of tumor cells through the $\mathrm{LN}$ capsule may reflect the aggressiveness acquired from a clone deriving from the primary tumor or occurring within the metastatic nodes [17]. Therefore, several authors have proposed the detection and quantification of ENE in the surgical specimen as a novel diagnostic tool for a correct assessment of tumor staging and for a personalization of adjuvant therapy [16]. However, no information is available on the molecular mechanisms underpinning the ENE phenotype. Thus, the main aim of this work was the investigation of the heterogeneous intratumor molecular landscape and immune-microenvironment in a series of ENE-positive CRCs.

\section{Materials and methods}

\section{Patients and samples}

A series of consecutive 65 TNM stages III $(n=45)$ and IV $(n=20)$ formalin-fixed paraffin embedded (FFPE) primary CRC and their matched LNs were retrieved from the archives of the Surgical Pathology Unit of the Padua University (samples collected in year 2016). The study was approved by the local Ethic Committee (n. 0032705/16).

Patients who had received neoadjuvant chemo-radiotherapy, patients under the age of 20 , and cases with a number of histologically examined LNs lower than 12 were excluded from the selection.

Two gastrointestinal pathologists, who were unaware of any clinical information, re-evaluated all the original haematoxylin and eosin stained slides according to the 2010 World Health Organization classification [18]. The presence of ENE was defined as an extension of tumor cells through the nodal capsule into the perinodal adipose tissue [5] and it was assessed as present or absent. Tumor cell nodules in perivisceral adipose tissue, which are not connected with the primary tumor and are not surrounded by lymphoid tissue, were excluded due to a consideration of them as tumor deposits.

Only cases with adequate material for the subsequent molecular characterization were selected. In each case three different matched samples were obtained: (i) the primary CRC; (ii) a metastatic LN with no extranodal extension of the tumor (i.e. ENE-negative LNs were entirely examined by serial sections to exclude the present of extra-nodal involvement); (iii) an ENE positive metastatic LN.

A total of 26 cases resulted ENE positive, but adequate material was available only for $22(\mathrm{M} / \mathrm{F}=15 / 7$; age $70.2 \pm 13.7$, range $40-89$; grading $\mathrm{G} 2=4, \mathrm{G} 3=18$; staging IIIb $=2$, IIIc $=14$, IV =6; all Caucasian; DNA mismatch repair machinery deficient tumors $[\mathrm{MMRd}]=4$, all with defects in MLH1/PMS2 expression). Thus, a total of 66 samples (i.e. three per case) were selected and further analysed.

\section{Immunohistochemistry}

Immunohistochemical stains for PD-L1 (clone 22C3; Dako, Glostrup, Denmark), CD4 (Dako), CD8 (Dako), CD68 (Dako), CD80 (Novus Biologicals, Littleton, CO), MLH1 (ES05; Dako), MSH2 (clone FE11; Dako), MSH6 (clone EP49; Dako), and PMS2 (clone EP51; Dako) were automatically performed in 3-4 $\mu \mathrm{m}$ sections using the Bond Polymer Refine Detection kit (Leica Biosystems, Newcastle upon Tyne, UK) in the BOND-MAX system (Leica Biosystems). Appropriate positive and negative controls were run concurrently.

PD-L1 expression was assessed both in neoplastic epithelia and in infiltrating lymphocytes. The positive neoplastic cells percentage was evaluated jointly by two pathologists. For epithelial immunoreactivity, only moderate/strong membranous staining up to $1 \%$ was considered positive. Stromal positive lymphoid cells were 
(See figure on next page.)

Fig. 1 a Immunohistochemical characterization of 22 ENE-positive colorectal carcinomas. In each case, stromal positive lymphoid T cells were evaluated in five high-power fields $(40 \times)$ in different areas of the samples: (i) central part of the primary CRC; (ii) invasive front of the primary CRC; (iii) intratumoral infiltration of the metastatic LN sample; (iv) intratumoral infiltration of the ENE positive LN sample; (v) invasive front of the ENE positive LN sample. A significantly higher percentage of CD4-, CD8- and CD68-positive cells was observed at the invasive front of both CRCs and in ENE in contrast with what observed at the core of both CRCs and their matched nodal metastases. ENE was also characterized by a significantly higher number of CD80-positive cells. $\left.{ }^{*} p<0.05 ;{ }^{* *} p<0.01 ;{ }^{* * *} p<0.001\right)$. b Representative images of CD80-positive cells distribution within the considered samples (original magnifications $20 \times$ and $40 x$; scale bars $=100 \mu \mathrm{m}$ )

evaluated in five HPF $(40 \times)$ in different areas of the samples: (i) central part of the primary CRC; (ii) invasive front of the primary CRC; (iii) intratumoral infiltration of the metastatic LN sample; (iv) intratumoral infiltration of the ENE positive LN sample; (v) invasive front of the ENE positive LN sample (Fig. 1). Presence of lymph node-like structures at the edge of the primary tumors were not considered in the analysis. Stromal PD-L1 positive cells infiltration of tumors was assessed by semiquantitative estimation of the density of PD-L1-positive cells, and was scored as 0 , no positive cell; (1) sporadic PD-L1 ${ }^{+}$cells; (2) moderate numbers of PD-L1 ${ }^{+}$cells; (3) abundant occurrence of PD-L1 ${ }^{+}$cells.

The infiltrating CD4, CD8, CD68 and CD80 leukocytes were counted in five HPF of the previously mentioned five randomly selected tumor areas and final counts were normalized within the same case.

DNA mismatch repair machinery deficient tumors (MMRd) were defined by the absence of nuclear staining in one of the two heterodimers MLH1/PMS2 and/or MSH2/MSH6 in tumor cells, as assessed in the colorectal setting [19].

\section{Hotspot multigene mutational profiling}

DNA was obtained from the FFPE samples after enrichment for neoplastic cells, as previously described with minor modifications [20]. Suitable areas for microdissection were selected from the archival haematoxylin and eosin slides and the corresponding tissue blocks were serially cut to $10-\mu \mathrm{m}$-thin sections. Unstained sections were, subsequently, deparaffinized and slightly counterstained with haematoxylin. Tumor cells were dissected manually using a sterile syringe needle, and at least $60 \%$ of neoplastic cells were collected from the primary carcinoma and two different metastatic lymph-node, one of which characterized by the presence of ENE. In the ENEpositive lymph-node, only the region with extracapsular extension was considered for microdissection. DNA was extracted using the QIAamp DNA formalin-fixed, paraffin-embedded tissue kit (Qiagen, Milan, Italy) and qualified as previously performed [21].

DNA obtained from the microdissected tumor components underwent hotspot multigene mutational profiling including 164 hotspot regions of the $A K T 1, A P C$,
BRAF, CTNNB1, KIT, KRAS, NRAS, PDGFRA, PIK3CA, PTEN and TP53 genes using a custom panel (Diatech Pharmacogenetics, Jesi, Italy; primers and protocol available upon request) run on a MassARRAY Dx Analyzer 4 (Agena Bioscience, Hamburg, Germany) [22]. The observed somatic mutations were confirmed by Sanger sequencing (Applied Biosystems 3130xl Genetic Analyser; Life Technologies, Monza, Italy).

\section{Statistical analysis}

The strength of the association between the different histological lesions and the immunohistochemical/molecular features was calculated by applying the Wilcoxon signed-rank test and the Mann-Whitney test, as appropriate. Stata software (Stata Corporation, College Station, TX) was used for all calculations. A $p$ value $<0.05$ was considered significant.

\section{Results}

ENE phenotype is characterized by an enhanced inflammatory response at the invasive front

A series of 22 ENE-positive CRCs were analysed by immunohistochemistry for PD-L1, CD4, CD8, CD68 and CD80 expression. For each case, three different samples were considered and analysed: the primary CRC, an ENE-negative and an ENE-positive metastatic LN.

Immunohistochemical analyses showed a significantly higher percentage of CD4-, CD8- and CD68-positive cells at the CRC and ENE invasive fronts in comparison with what observed at the core of both CRCs and their matched ENE-negative nodal metastases (Fig. 1a).

For CD4, the normalized number of positive T-cells was 1.82-fold higher in the CRC invasive front in comparison of the number of positive cells observed at the center of the tumor ( $p=0.0026$; paired $t$ test) and 1.71 in ENE invasive front in comparison to the matched ENEnegative metastatic LNs ( $p=0.029$; paired $t$-test).

For CD8, the normalized number of positive T-cells was 3.04-fold higher in the CRC invasive front in comparison of the number of positive cells observed at the center of the tumor $(p<0.0001$; paired $t$-test) and 1.74 in ENE invasive front in comparison to the matched ENEnegative metastatic LNs ( $p=0.008$; paired $t$-test). 

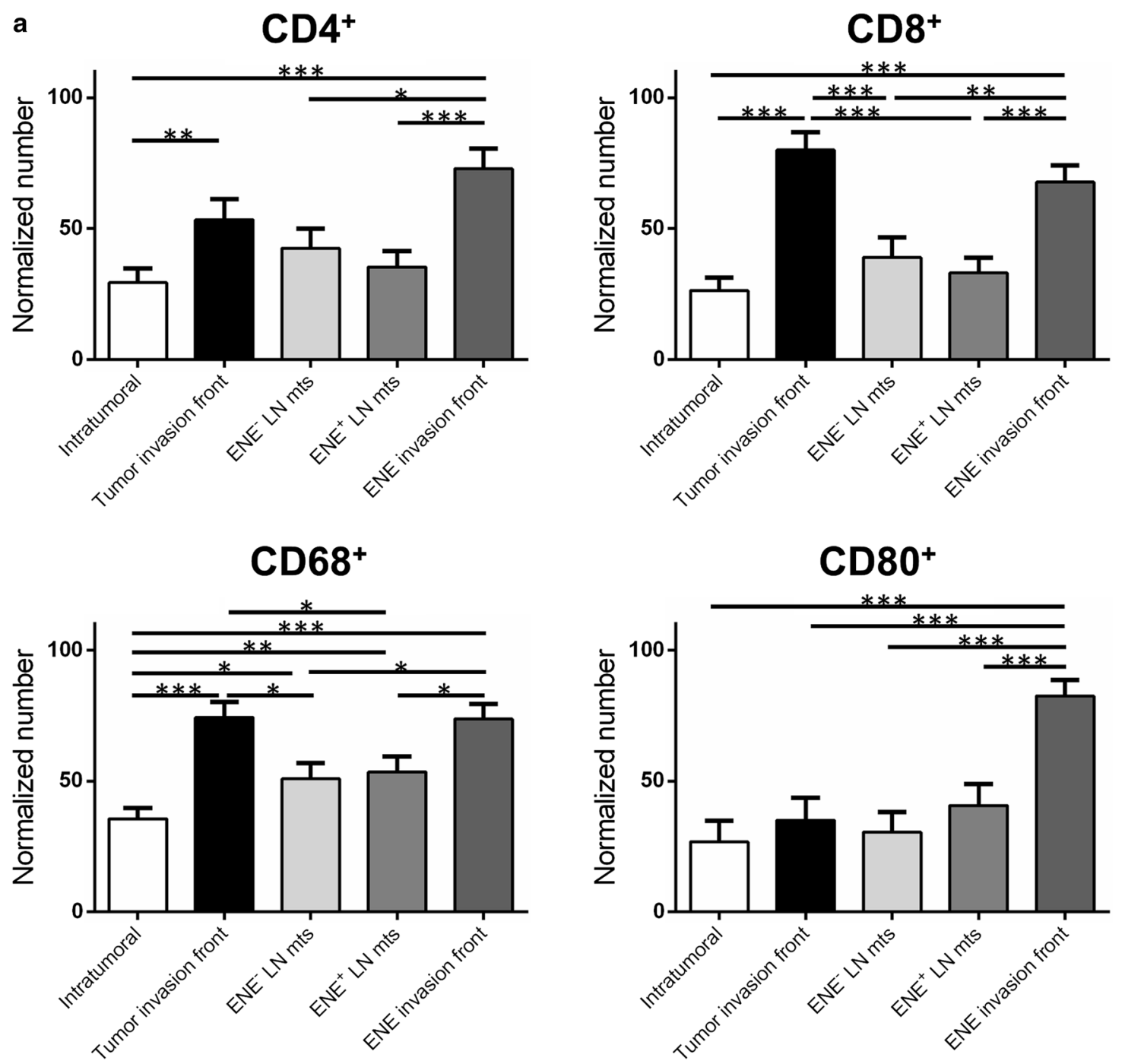

b Primary tumor

LN metastases

Intratumoral Invasive front

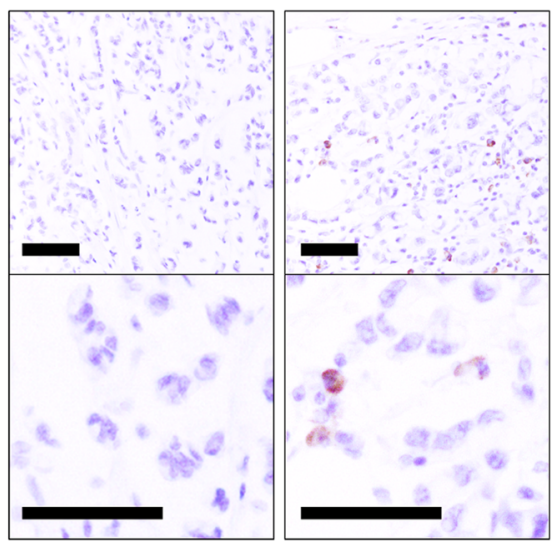

ENE-

$\mathrm{ENE}^{+}$

$\mathrm{ENE}^{+}$

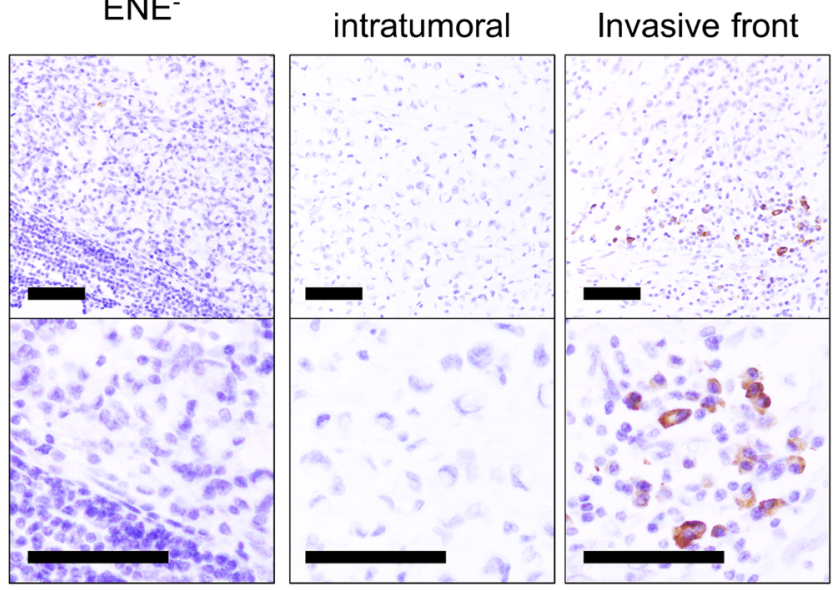


For CD68, the normalized number of positive macrophages was 2.08-fold higher in the CRC invasive front in comparison of the number of positive cells observed at the center of the tumor $(p<0.0001$; paired $t$-test) and 1.45 in ENE invasive front in comparison to the matched ENE-negative LNs ( $p=0.015$; paired $t$-test).

A significantly higher prevalence of CD80-positive cells was detected in the ENE samples only (Fig. 1a, b). In particular, the normalized number of positive cells was 2.70fold higher in ENE invasive front in comparison to the matched ENE-negative LNs ( $p<0.001$; paired $t$-test).

No significant number of epithelial, or leukocytic PD-L1 positive cells was observed among the different considered samples.

The four cases with MMRd presented a relatively, but not significant, higher number of CD4 and CD8 infiltrating cells (in both primary and metastatic LNs) in comparison to MMR proficient (MMRp) cases, and were also characterised by a higher number of $\mathrm{CD} 4 / \mathrm{CD} 8$ positive cells at both the CRC invasive front in comparison to the center of the tumor and in ENE invasive front in comparison to the matched ENE-negative metastatic LNs.

\section{No specific mutational signature characterizes the ENE phenotype}

Fifteen out of the original series of 22 cases were further profiled through a hotspot multigene mutational custom panel.

In primary tumors, a total of 25 somatic mutations were observed among the 11 cancer-related genes (Fig. 2). In 14 of 15 (93\%) primary CRC samples, at least one somatic mutation was detected; eight lesions were found to have multiple driver gene somatic mutations.

The TP53 gene was the most frequently mutated gene (8 out of 15), followed by $A P C$ (six cases) and $B R A F$ (four cases; all MMRd). KRAS, NRAS and PIK3CA were mutated in two cases at most. In 11 out of 15 (73\%), the molecular profiling of the primary CRC was consistent with that of its matched metastatic LNs, whereas a discrepancy between the mutational profiling of the primary CRC and its matched metastatic LNs was found in four cases (Fig. 2).

The detected allele frequencies of the mutations observed in the four discordant cases was consistent with either polyclonal expansion or monoclonal proliferation of the disease (intra-tumor heterogeneity) in line with recent evidence suggesting that nodal dissemination from primary CRCs is not necessarily related to genetic bottlenecks [23].

No significant association was observed between a peculiar mutational profile and an increase/decrease in inflammatory infiltration.

\section{Discussion}

For many years, the interest in oncological research has focused on understanding the mechanisms that would induce the acquisition of an aggressive phenotype by the neoplastic cells. This may represent the biological rationale in the development of new therapeutics which, on one side, would impair the metastatic ability and, on the other, personalize the oncological and surgical managements of the disease.

Worldwide, CRC is a major cause of morbidity and mortality $[1,2]$. The TNM staging system represents the best patient's prognostic factor at time of the diagnosis. Although the TNM staging system is constantly updated, outcomes of patients with similar histological features falling within the same risk category may have heterogeneous outcomes (i.e. pT3pN1 versus pT4 and/or pN2 in Stage III CRC) thus impacting on clinical decisions [24]. It is, therefore, clear that there is an unmet need to identify new prognostic factors to better stratify the patients, both from a histological and molecular point of view.

The detection of ENE has been demonstrated to be an important prognostic factor in many epithelial cancers [3-14]. Indeed, the latest TNM edition has highlighted the need to report the presence of ENE in lymph node metastasis in case of the head and neck tumors and the vulvar squamous cell carcinoma $[25,26]$.

Several lines of evidence have proven that, even in CRC, the presence of ENE correlates with a patient's poor prognosis both in terms of risk of recurrence and overall survival $[4,7,27-30]$.

Despite the recognized prognostic impact of the ENE phenotype, no previous work has tried to elucidate its molecular landscape. One of the bottlenecks in this field is the (almost) mandatory use of formalin-fixed paraffinembedded (FFPE) specimens, which potentially affects many down-stream molecular pathology analyses [31]. Moreover, most of the lesions are small and, thus, their comprehensive molecular characterization is technically challenging.

Starting from archival FFPE material, we investigated: (i) the ENE-associated inflammatory infiltrate by immunohistochemistry; (ii) the ENE molecular background by applying a FFPE-friendly custom panel to characterize 164 hotspot regions of CCR-related genes. Of the initial series of 65 consecutive TNM stages III and IV primary CRC and their matched metastatic LNs, only 22 presented adequate material for the subsequent molecular characterization.

The immunohistochemical evaluation for the presence of tumor-infiltrating T lymphocytes (CD4 and CD8) and macrophages (CD68) demonstrated a significantly higher prevalence of inflammatory infiltrate at the neoplastic invasive front of the primary tumor and of the ENE in 


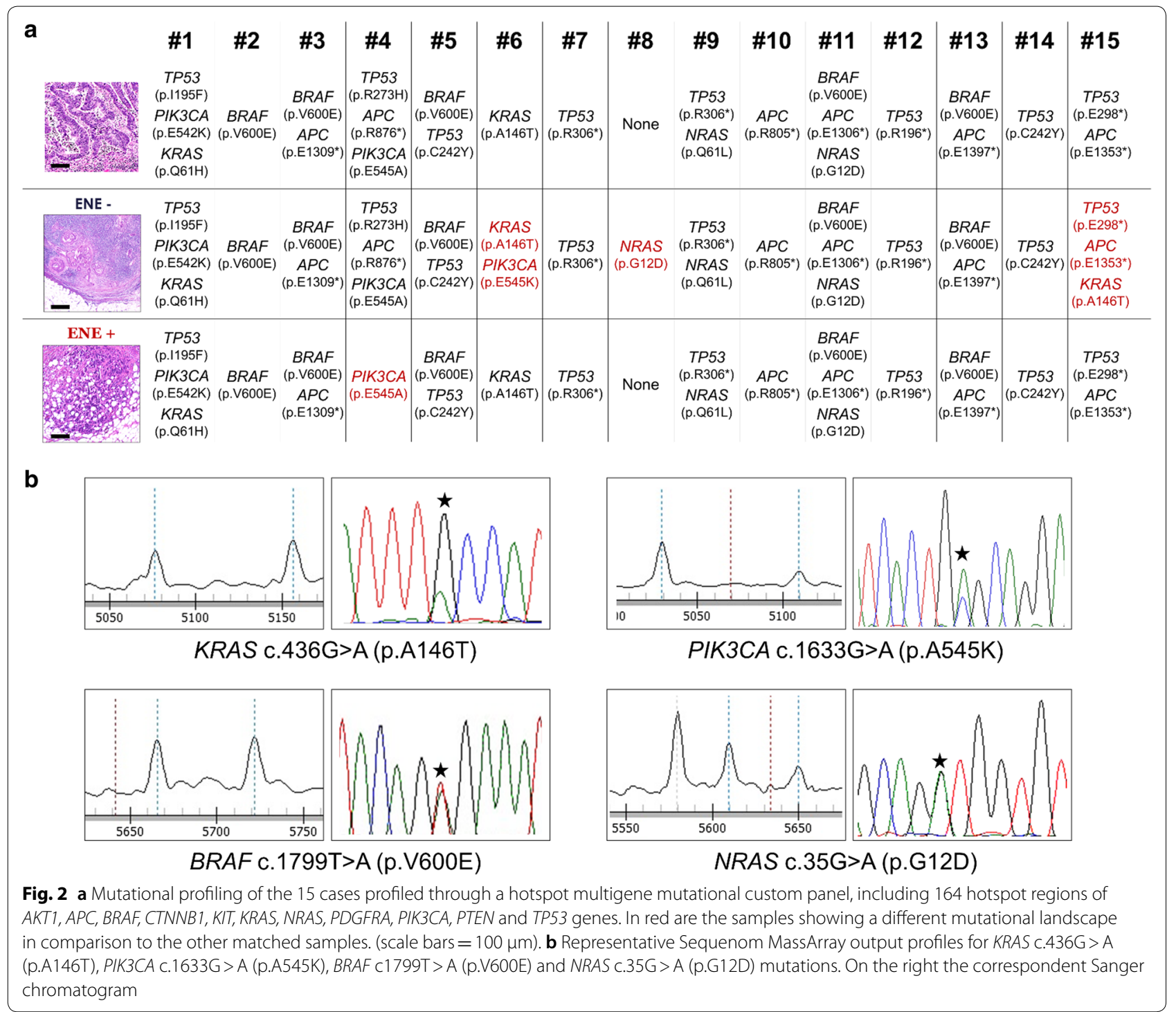

contrast with what observed at the core of both CRCs and their matched intra-nodal metastases. These data suggest that there is a common host-related inflammatory systemic response against the infiltrating neoplastic cells. An important negative aspect of this study is the lack of a comprehensive tumor immune microenvironment characterization, which was not possible due to the limited amount of (FFPE) material. Further expression profiling studies should investigate the role of the different tumor areas/tumor-associated stroma in the polarization of the monocyte-macrophage lineage and of the activation of an antitumor milieu.

Of note, high prevalence of CD80 positive cells was observed at the ENE invasive front. This protein is present on dendritic cells, activated B cells and monocytes and its overexpression provides a costimulatory signal necessary for $\mathrm{T}$ cell activation and survival. A significant overexpression of CD80 in colonic mucosa has been found in early stages of colorectal carcinogenesis and was demonstrated to play a role in immune surveillance mechanisms in the transition from low-grade to high-grade dysplasia [32, 33]. However, a strong CD80 expression has been associated to resistance to adjuvant chemotherapy suggesting the failure of this specific immune surveillance mechanism in advanced stage of digestive tract cancer [34]. Thus, the association of ENE status with an overexpression of CD80 may be one of the factors which could potentially explain the more aggressive biological behaviour displayed by metastatic CRCs with ENE [16]. The results of this exploratory study 
call for further investigations about the immunological microenvironment of metastatic lymph nodes with ENE, also for addressing new potential therapeutic strategies.

Even on a relatively small series of cases and by analyzing a limited panel of CRC-associated genes, our study lack to demonstrate any clonal ENE-associated nodal metastases specific molecular driver. Indeed, $73 \%$ of the cases show that the mutations which were found in the primary tumors are consistent with those observed in their matched lymph node metastases demonstrating that the tumor clones are still the same (observed for both ENE positive and ENE negative ones). Moreover, no difference was found between the ENE-associated nodal metastases and the ENE-negative one or the correspondent primary tumor in the same patient. This lack of association may be explained with the small sample size of our study, but it is more likely that somatic mutations are not a driver mechanism in the acquisition of the ENE phenotype in CRCs. Recent reports have demonstrated an important clustering of cancer stem-like cells at the CRC invasive front and their important role in the development of tumor heterogeneity [35-38]. In the CRC setting, CD44 variant-positive cancer stem cells have been demonstrated to be resistant to redox stress in the tumor microenvironment by enhancing the ESRP1CD44v-xCT-GSH (cysteine/glutamate antiporter) axis $[35,38,39]$, and thus these cells are almost unaffected by selective pressure determined by oxygen deprivation and anti-cancer treatments Another interaction involving beta-catenin in CRC regards CD200, a membrane protein that characterizes neoplastic cells with cancer stem properties [40]. Due to the important role of CD200 in regulating tumor microenvironment in this setting, its expression may be of importance also in ENE-positive CRCs. Future studies are therefore needed to better define the complete profiles of ENE-positive CRC cell clones.

With our study, we highlight that the biological mechanisms on the basis of ENE in CRCs should be further investigated in the complex immunological microenvironment rather than in the genetic profiles of metastatic cells, including CD44 and CD200. It is also of importance that in other solid malignancies an ENE-specific molecular profile has been detected by comparing ENE-negative and ENE-positive cases [41], but in CRC the importance of metastases microenvironment may be more significant.

\section{Conclusion}

Overall our data demonstrated that there is, on one side, no specific molecular features based on our relatively limited genotypic study for the acquisition of the ENE phenotype but, on the other, the overexpression of CD80 and
T lymphocytes/macrophages infiltration at the ENE invasive front may suggest further investigations on a possible application of novel immunotherapeutic approaches in ENE-positive CRCs.

\begin{abstract}
Abbreviations
CRC: colorectal cancer; ENE: extranodal extension; FFPE: formalin-fixed and paraffin-embedded; MMRd: mismatch repair deficient; MMRp: mismatch repair proficient; TNM: tumor-node-metastasis.
\end{abstract}

\section{Authors' contributions}

$M F, L V, D S, N V, C L$, and MR conceived and planned the experiments; MF, LV, $D S, G N F, G M, C L, R S, N V, M A$, and EDA carried out the experiments; MF, MS, RC, $\mathrm{FL}, \mathrm{CM}, \mathrm{NV}, \mathrm{MA}, \mathrm{SL}, \mathrm{SP}$, and MR collected and qualified the samples; MF, LV, DS, $M S, F L, N V, M A, N V, C L$, and $M R$ contributed to the interpretation of the results; $M F, L V, D S, C L$, and $M R$ took the lead in writing the manuscript. All the authors provided critical feedback and helped the research, analysis and manuscript. All authors read and approved the final manuscript.

\section{Author details}

${ }^{1}$ Surgical Pathology \& Cytopathology Unit, Department of Medicine (DIMED), University of Padua, via Gabelli 61, 35121 Padua, Italy. ${ }^{2}$ Department of Surgical Oncology and Gastroenterology (DiSCOG), University of Padua, Padua, PD,

Italy. ${ }^{3}$ Unit of Oncology 1, Department of Clinical and Experimental Oncology, Istituto Oncologico Veneto, IOV-IRCCS, Padua, PD, Italy. ${ }^{4}$ Division of Molecular Pathology, The Institute of Cancer Research, Sutton, London, UK. ${ }^{5}$ Department of Medicine, The Royal Marsden NHS Trust, Sutton, London, UK. ${ }^{6}$ Nanoinspired Biomedicine Laboratory, Institute of Pediatric Research, Fondazione Città della Speranza, Padua, PD, Italy. ${ }^{7}$ Department of Nanomedicine, The Methodist Hospital Research Institute, Houston, TX, USA. ${ }^{8}$ National Research Council, Neuroscience Institute, Aging Branch, Padua, PD, Italy. ${ }^{9}$ Department of Diagnostics and Public Health, Section of Pathology, University and Hospital Trust of Verona, Verona, VR, Italy. ${ }^{10}$ Veneto Cancer Registry, Padua, PD, Italy.

${ }^{11}$ National Institute of Gastroenterology-Research Hospital, IRCCS "S. de Bellis", 70013 Castellana Grotte, BA, Italy.

\section{Acknowledgements}

Not applicable.

Competing interests

The authors declare that they have no competing interests.

Availability of data and materials

All the data and material could be traced from the paper or can be requested to the corresponding author.

\section{Consent for publication}

All the listed authors have participated in the study, and have seen and approved the submitted manuscript.

Ethics approval and consent to participate

The study was approved by the local Ethic Committee (n. 0032705/16).

Funding

This work was partly supported by a grant from the Italian Association for Cancer Research (AIRC Regional Grant 2008 N. 6421).

\section{Publisher's Note}

Springer Nature remains neutral with regard to jurisdictional claims in published maps and institutional affiliations.

Received: 23 July 2018 Accepted: 3 September 2018

Published online: 06 September 2018 


\section{References}

1. Arnold M, Sierra MS, Laversanne M, Soerjomataram I, Jemal A, Bray F. Global patterns and trends in colorectal cancer incidence and mortality. Gut. 2017;66:683-91.

2. Brenner H, Kloor M, Pox CP. Colorectal cancer. Lancet. 2014;383:1490-502.

3. Luchini C, Veronese N, Pea A, Sergi G, Manzato E, Nottegar A, Solmi M, Capelli P, Scarpa A. Extranodal extension in N1-adenocarcinoma of the pancreas and papilla of Vater: a systematic review and meta-analysis of its prognostic significance. Eur J Gastroenterol Hepatol. 2016;28:205-9.

4. Luchini C, Nottegar A, Pea A, Solmi M, Stubbs B, Capelli P, Sergi G, Manzato E, Fassan M, Wood LD, Scarpa A, Veronese N. Extranodal extension is an important prognostic parameter for both colonic and rectal cancer. Ann Oncol. 2016:27:955-6.

5. Luchini C, Wood LD, Cheng L, Nottegar A, Stubbs B, Solmi M, Capelli P, Pea A, Sergi G, Manzato E, Fassan M, Bagante F, Bollschweiler E, Giacopuzzi S, Kaneko T, de Manzoni G, Barbareschi M, Scarpa A, Veronese N. Extranodal extension of lymph node metastasis is a marker of poor prognosis in oesophageal cancer: a systematic review with meta-analysis. J Clin Pathol. 2016. https://doi.org/10.1136/jclinpath-2016-203830.

6. Luchini C, Nottegar A, Solmi M, Sergi G, Manzato E, Capelli P, Scarpa A, Veronese N. Prognostic implications of extranodal extension in nodepositive squamous cell carcinoma of the vulva: a systematic review and meta-analysis. Surg Oncol. 2016;25:60-5.

7. Luchini C, Nottegar A, Pea A, Solmi M, Stubbs B, Capelli P, Sergi G, Manzato E, Fassan M, Wood LD, Scarpa A, Veronese N. Significance of the prognostic stratification of extranodal extension in colorectal cancer. Ann Oncol. 2016;27:1647

8. Luchini C, Fleischmann A, Boormans JL, Fassan M, Nottegar A, Lucato $P$ Stubbs B, Solmi M, Porcaro A, Veronese N, Brunelli M, Scarpa A, Cheng $L$. Extranodal extension of lymph node metastasis influences recurrence in prostate cancer: a systematic review and meta-analysis. Sci Rep. 2017;7:2374.

9. Luchini $\mathrm{C}$, Veronese $\mathrm{N}$. The importance of extranodal extension in metastatic head and neck squamous cell carcinoma, in the light of the new AJCC cancer staging system. Oral Oncol. 2017;66:e1-2.

10. Nottegar A, Veronese $N$, Senthil M, Roumen RM, Stubbs B, Choi AH, Verheuvel NC, Solmi M, Pea A, Capelli P, Fassan M, Sergi G, Manzato E, Maruzzo M, Bagante F, Koc M, Eryilmaz MA, Bria E, Carbognin L, Bonetti F, Barbareschi M, Luchini C. Extra-nodal extension of sentinel lymph node metastasis is a marker of poor prognosis in breast cance patients: a systematic review and an exploratory meta-analysis. Eur J Surg Oncol. 2016:42:919-25.

11. Veronese N, Luchini C, Nottegar A, Kaneko T, Sergi G, Manzato E, Solmi M, Scarpa A. Prognostic impact of extra-nodal extension in thyroid cancer: a meta-analysis. J Surg Oncol. 2015;112:828-33.

12. Veronese N, Fassan M, Wood LD, Stubbs B, Solmi M, Capelli P, Pea A, Nottegar A, Sergi G, Manzato E, Carraro S, Maruzzo M, Cataldo I, Bagante F, Barbareschi M, Cheng L, Bencivenga M, de Manzoni G, Luchini C. Extranodal extension of nodal metastases is a poor prognostic indicator in gastric cancer: a systematic review and meta-analysis. J Gastrointest Surg. 2016;20:1692-8.

13. Luchini C Veronese $N$. Extranodal extension of nodal metastasis is the main prognostic moderator in squamous cell carcinoma of the esophagus after neoadjuvant chemoradiotherapy. J Thorac Dis. 2017:9:3609-12.

14. Luchini C, Veronese N, Nottegar A, Cheng M, Kaneko T, Pilati C, Tabbo F, Stubbs B, Pea A, Bagante F, Demurtas J, Fassan M, Infante M, Cheng L, Scarpa A. Extranodal extension of nodal metastases is a poor prognostic moderator in non-small cell lung cancer: a meta-analysis. Virchows Arch. 2018;472:939-47

15. Wittekind C, Gospodarowicz MK, Sobin LH. TNM classification of malignant tumours. Chichester: Wiley-Blackwell; 2009.

16. Veronese N, Nottegar A, Pea A, Solmi M, Stubbs B, Capelli P, Sergi G, Manzato E, Fassan M, Wood LD, Scarpa A, Luchini C. Prognostic impact and implications of extracapsular lymph node involvement in colorectal cancer: a systematic review with meta-analysis. Ann Oncol. 2016;27:42-8.

17. Stitzenberg KB, Meyer AA, Stern SL, Cance WG, Calvo BF, Klauber-DeMore N, Kim HJ, Sansbury L, Ollila DW. Extracapsular extension of the sentinel lymph node metastasis: a predictor of non sentinel node tumor burden. Ann Surg. 2003;237:607-12 (discussion 612-3).
18. Bosman FT, Carneiro F, Hruban RH, Theise ND. World Health Organization classification of tumours of the digestive system. Geneva: World Health Organization; 2010

19. Remo A, Fassan M, Lanza G. Immunohistochemical evaluation of mismatch repair proteins in colorectal carcinoma: the AIFEG/GIPAD proposal. Pathologica. 2016;108:104-9.

20. Bellan A, Cappellesso R, Lo Mele M, Peraro L, Balsamo L, Lanza C, Fassan M, Rugge M. Early signet ring cell carcinoma arising from colonic adenoma: the molecular profiling supports the adenoma-carcinoma sequence. Hum Pathol. 2016;50:183-6.

21. Simbolo M, Gottardi M, Corbo V, Fassan M, Mafficini A, Malpeli G, Lawlor RT, Scarpa A. DNA qualification workflow for next generation sequencing of histopathological samples. PLoS ONE. 2013;8:e62692.

22. Galuppini F, Salmaso R, Valentini E, Lanza C, Maretto I, Nitti D, Rugge M, Fassan M. Adenosquamous gallbladder carcinoma: multigene hotspot mutational profiling reveals a monoclonal origin of the two components. Pathol Res Pract. 2017;213:1010-3.

23. Gerlinger M. Metastasis seeding cells: lone invaders or mass migrators? Clin Cancer Res. 2018;24:2032-4

24. Grothey A, Sobrero AF, Shields AF, Yoshino T, Paul J, Taieb J, Souglakos J, Shi Q, Kerr R, Labianca R, Meyerhardt JA, Vernerey D, Yamanaka T, Boukovinas I, Meyers JP, Renfro LA, Niedzwiecki D, Watanabe T, Torri V, Saunders M, Sargent DJ, Andre T, Iveson T. Duration of adjuvant chemotherapy for stage III colon cancer. N Engl J Med. 2018;378:1177-88.

25. Huang SH, O'Sullivan B. Overview of the 8th edition TNM classification for head and neck cancer. Curr Treat Options Oncol. 2017;18:40.

26. van der Velden J, van Lindert AC, Lammes FB, Kate FJT, Sie-Go DM, Oosting H, Heintz AP. Extracapsular growth of lymph node metastases in squamous cell carcinoma of the vulva. The impact on recurrence and survival. Cancer. 1995;75:2885-90.

27. Brabender J, Bollschweiler E, Holscher AH, Strobel K, Gutschow C, Prenzel K, Grimminger P, Drebber U, Schroder W, Metzger R, Vallbohmer D. The prognostic impact of extracapsular lymph node involvement in rectal cancer patients: implications for staging and adjuvant treatment strategies. Oncol Lett. 2012;3:825-30.

28. Fujii T, Tabe Y, Yajima R, Yamaguchi S, Tsutsumi S, Asao T, Kuwano H. Extracapsular invasion as a risk factor for disease recurrence in colorectal cancer. World J Gastroenterol. 2011;17:2003-6.

29. Heide J, Krull A, Berger J. Extracapsular spread of nodal metastasis as a prognostic factor in rectal cancer. Int J Radiat Oncol Biol Phys. 2004;58:773-8

30. Kim CW, Kim J, Yeom SS, Lee JL, Yoon YS, Park IJ, Lim SB, Baek S, Yu CS, Kim JC. Extranodal extension status is a powerful prognostic factor in stage III colorectal cancer. Oncotarget. 2017;8:61393-403.

31. Fassan M, Baffa R, Kiss A. Advanced precancerous lesions within the Gl tract: the molecular background. Best Pract Res Clin Gastroenterol. 2013;27:159-69.

32. Scarpa M, Behboo R, Angriman I, Cecchetto A, D'Inca R, Termini B, Barollo M, Ruffolo C, Polese L, Sturniolo GC, D'Amico DF. Expression of costimulatory molecule CD80 in colonic dysplasia in ulcerative colitis: an immunosurveillance mechanism against colorectal cancer? Int J Colorectal Dis. 2006;21:776-83.

33. Scarpa M, Brun P, Scarpa M, Morgan S, Porzionato A, Kotsafti A, Bortolami M, Buda A, D'Inca R, Macchi V, Sturniolo GC, Rugge M, Bardini R, Castagliuolo I, Angriman I, Castoro C. CD80-CD28 signaling controls the progression of inflammatory colorectal carcinogenesis. Oncotarget. 2015:6:20058-69.

34. Ghidini M, Cascione L, Carotenuto P, Lampis A, Trevisani F, Previdi MC, Hahne JC, Said-Huntingford I, Raj M, Zerbi A, Mescoli C, Cillo U, Rugge M, Roncalli M, Torzilli G, Rimassa L, Santoro A, Valeri N, Fassan M, Braconi C. Characterisation of the immune-related transcriptome in resected biliary tract cancers. Eur J Cancer. 2017;86:158-65.

35. Yoshida GJ. The heterogeneity of cancer stem-like cells at the invasive front. Cancer Cell Int. 2017;17:23.

36. Yoshida GJ, Saya H. Therapeutic strategies targeting cancer stem cells. Cancer Sci. 2016;107:5-11.

37. Yoshida GJ. Metabolic reprogramming: the emerging concept and associated therapeutic strategies. J Exp Clin Cancer Res. 2015;34:111.

38. Yoshida GJ. Emerging roles of Myc in stem cell biology and novel tumor therapies. J Exp Clin Cancer Res. 2018:37:173. 
39. Yoshida GJ, Saya H. Inversed relationship between CD44 variant and c-Myc due to oxidative stress-induced canonical Wnt activation. Biochem Biophys Res Commun. 2014;443:622-7.

40. Zhang SS, Huang ZW, Li LX, Fu JJ, Xiao B. Identification of CD200+ colorectal cancer stem cells and their gene expression profile. Oncol Rep. 2016;36:2252-60
41. Wang W, Lim WK, Leong HS, Chong FT, Lim TK, Tan DS, Teh BT, lyer NG. An eleven gene molecular signature for extra-capsular spread in oral squamous cell carcinoma serves as a prognosticator of outcome in patients without nodal metastases. Oral Oncol. 2015;51:355-62.
Ready to submit your research? Choose BMC and benefit from:

- fast, convenient online submission

- thorough peer review by experienced researchers in your field

- rapid publication on acceptance

- support for research data, including large and complex data types

- gold Open Access which fosters wider collaboration and increased citations

- maximum visibility for your research: over 100M website views per year

At BMC, research is always in progress.

Learn more biomedcentral.com/submissions 